\title{
Data Communication of Process Control System for Plate Mill
}

\author{
Zhijie Jiao ${ }^{1,}$,, Xiaotong Meng ${ }^{1, b}$, Chunyu $\mathrm{He}^{1, \mathrm{c}}$ and Jun Wang ${ }^{1, \mathrm{~d}}$ \\ ${ }^{1}$ State Key Lab of Rolling and Automation, Northeastern University, Shenyang 110819, China \\ ajiaozj@163.com
}

Keywords: Plate mill, Process control system, Data communication

Abstract. For plate mill, data communication between Process Control System (PCS) and other system are built with different communication methods. The communication between PCS and Basic Automation system (BAS) is based on the industry Ethernet. Every $200 \mathrm{~ms}$, BAS sends actual data to PCS, and PCS sends setup data to BAS according to the trigger events. The communication between PCS and Human Machine Interface (HMI) is based on OPC protocol, and data tags are used. Mediation tables are used for the database communication between PCS and Manufacturing Execution System (MES). Socket method is used for the plate mill PCS to communicate with furnace PCS, control cooling PCS and gauge meter system. All these communication methods have been used for the plate mill PCS on site.

\section{Introduction}

PCS is the most important part of the modern plate mill automation control system. With PCS, the rolling process can be tracked; model setup data is calculated and set. But the mill PCS must be assimilated into the whole automation system and the data communication is the base of the PCS functions. With data communication, the PCS can exchange information with other system [1]. Recently, some plate mill PCS are developed by the State Key Lab of rolling and automation, and the communication of PCS is running well.

\section{Process equipment and automation system of plate mill}

Process equipment. Plate mill line includes many process zones, and the typical double stands plate mill and the before and after process zones are shown in Fig.1. Before plate mill is the furnace, the slabs are heated to the target temperature. After discharging, the scale on the slab is flushed with descaling unit. Then plate is rolled with rough mill and finish mill to the target thickness. After rolling, plate is cooled with control cooling unit to the target temperature for the plate property controlling [2]. Instrumentation mounted on line is also shown in Fig.1. Hot metal detectors are used for plate detecting; pyrometers are used for plate temperature measurement and gauge meter is used for plate thickness measurement.

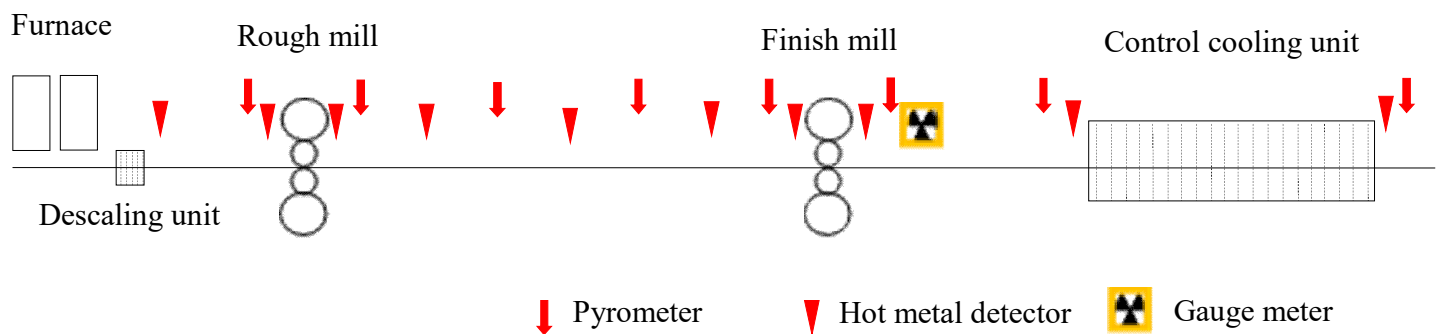

Fig. 1 Sketch of Plate mill line

Automation system. For the plate mill controlling, the automation system of plate mill is shown in Fig.2. L0 motor driving system, L1 BAS, L2 PCS and the HMI are included. The communication of mill PCS is also shown in Fig.2. The mill PCS is the core of communication, and the communication objects are includes BAS, HMI, MES and other systems such as furnace PCS, control cooling PCS and instrumentation. 


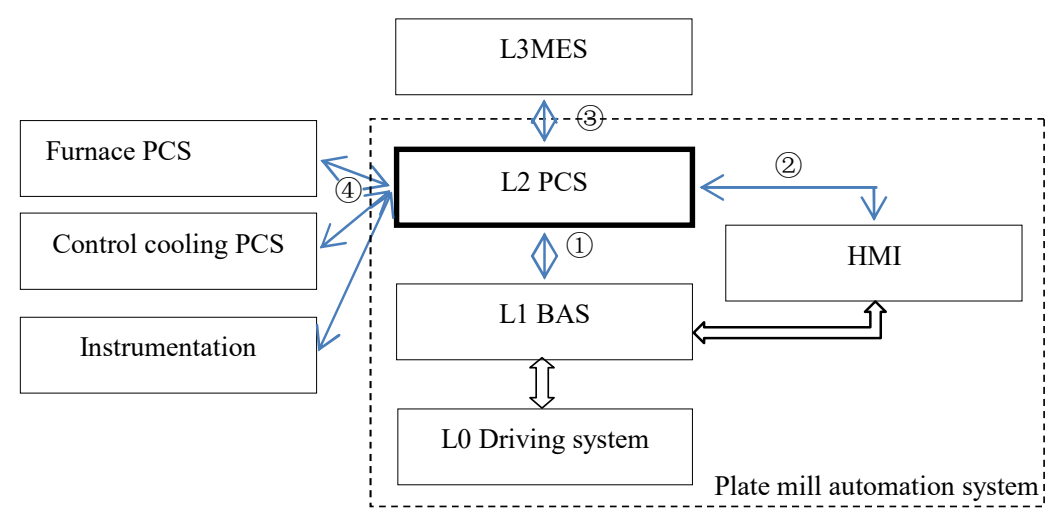

Fig.2 Sketch of Plate mill automation system

For one typical two stands plate mill, the hardware and software of automation system are provided. SIMENS TDC, S7-400 and S7-300 are used as BAS hardware, and HP DL580 PC servers are used as PCS and HMI hardware. The Windows operation system and Visual studio software are used for the PCS development.

\section{Data communication design and implementation}

The data communication of plate mill PCS is shown in Fig.2, these communications should be implemented: PCS communicates with BAS, HMI, MES, with before process zone furnace PCS, after process zone control cooling PCS and with gauge meter system. The detail realization is described following.

Communication with BAS. The communication information between PCS and BAS includes the actual rolling information sent by BAS and mill setup information sent by PCS. Because these information data is much, only part of Rough Mill (RM) data is listed in Table 1.

Table 1: Communication information between BAS and PCS

\begin{tabular}{|l|l|l|l|}
\hline Num. & Information items & Unit & \multirow{2}{*}{ Data flow } \\
\hline 1 & RM acutual current pass number & - & \\
\cline { 1 - 2 } 2 & RM actual rolling force of operation side & $\mathrm{kN}$ & \multirow{3}{*}{ BAS->PCS } \\
\hline 3 & RM actual roll gap of operation side & $\mathrm{mm}$ & \\
\hline 4 & RM actual upper roll speed & $\mathrm{RPM}$ & \\
\hline 5 & $\ldots \ldots$ & & \\
\hline 1 & RM set current pass number & - & \multirow{3}{*}{ PCS->BAS } \\
\hline 2 & RM set rolling force & $\mathrm{kN}$ & \\
\hline 3 & RM set bending force & $\mathrm{kN}$ & \\
\hline 4 & RM set roll gap & $\mathrm{mm}$ & \\
\hline 5 & $\ldots \ldots$ & & \\
\hline
\end{tabular}

The communication between PCS and BAS is based on the industry Ethernet. Because the SIMENS PLC is used as the BAS hardware, so the SIMATIC NET software is used for the communication[3]. The actual data sending from BAS to PCS is triggered by cycle timer, and the time period is set to $200 \mathrm{~ms}$. The setup data sending from PCS to BAS is triggered by event. PCS tracking function generates the event for the communication.

Communication with HMI. The communication information between PCS and HMI includes the operator intervention information and the HMI displaying information. Part of the Finishing Mill (FM) communication information is listed in Table 2. 
Table 2: Communication information between PCS and HMI

\begin{tabular}{|l|l|l|l|}
\hline Num. & Information items & Unit & Data flow \\
\hline 1 & FM rolling schedule calling trigger & - & \\
\cline { 1 - 3 } & FM plate ID & $\mathrm{kN}$ & \multirow{3}{*}{ HMI->PCS } \\
\hline 3 & FM slab thickness & $\mathrm{mm}$ & \\
\hline 4 & FM slab width & $\mathrm{mm}$ & \\
\hline 5 & $\ldots \ldots$ & & \\
\hline 1 & FM tracking update trigger & - & \multirow{2}{*}{ PCS->HMI } \\
\hline 2 & FM entry tracking plate ID1 & - & \\
\hline 3 & FM entry tracking plate ID2 & - & \\
\hline 4 & FM mill tracking plate ID & - & \\
\hline 5 & $\ldots \ldots$ & & \\
\cline { 1 - 2 }
\end{tabular}

The communication between PCS and HMI is based on the OPC (OLE for Process Control) protocol. The data tags are defined in HMI system, developed with SIMENS Wincc software. PCS can read and write the communication data tags directly. PCS reads data tags, and calls corresponding function according to the triggering tag. Then the HMI functions, such as computer schedule calling, roll data confirm, are implemented. PCS writes data tags for the information displaying on HMI. These information includes plate PDI, tracking information, model calculation information and PCS running information.

Communication with MES. The communication information between PCS and MES includes the production plan data sent by MES and the production report data sent by PCS. Part of this information data is list in Table 3.

Table 3: Communication information between PCS and MES

\begin{tabular}{|l|l|l|l|}
\hline Num. & Information items & Unit & Data flow \\
\hline 1 & Rolling order number & - & \\
\cline { 1 - 3 } & Slab number & - & \multirow{2}{*}{ MES->PCS } \\
\hline 3 & Slab actual thickness & $\mathrm{mm}$ & \\
\hline 4 & Slab actual width & $\mathrm{mm}$ & \\
\hline 5 & $\ldots \ldots$ & & \\
\hline 1 & Sending code number & - & \multirow{3}{*}{ PCS->MES } \\
\hline 2 & Plate number & - & \\
\hline 3 & Operation shift & - & \\
\hline 4 & Operation time & - & \\
\hline 5 & $\ldots \ldots$ & & \\
\hline
\end{tabular}

Communication with MES is based on the database. Through reading and writing data in database, the data communication can be realized. Though the communication speed is not so high, but this method has high stability and adaptability. It is especially suitable for the large amounts data but less demanding on speed communication between different system platforms[4]. The communication between PCS and MES is exactly satisfied this condition. In mediation database, the PDI and PDO tables are designed for the reading and writing data. The data from MES to PCS is written to PDI table by MES, and PCS reads data from this table according to the special flag. The data from PCS to MES is written to PDO table by PCS, and is read by MES. Communication between PCS and MES is shown in Fig.3.

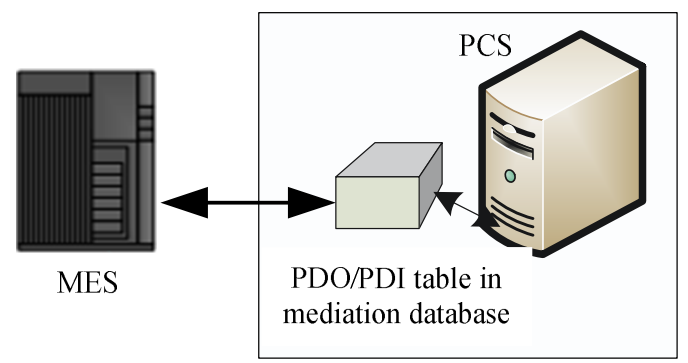

Fig. 3 Communication between PCS and MES 
Communication with other systems. Mill PCS also should communication with furnace PCS, control cooling PCS and gauge meter system. Part of this communication data is listed in Table 4.

Table 4: Communication information between PCS and other system

\begin{tabular}{|c|c|c|c|}
\hline Num. & Information Items & Unit & Data flow \\
\hline 1 & Slab number & - & \multirow{5}{*}{$\begin{array}{c}\text { Furnace } \\
\text { PCS -> Mill } \\
\text { PCS }\end{array}$} \\
\hline 2 & Slab discharge time & - & \\
\hline 3 & Slab in Furnace duration & $\mathrm{s}$ & \\
\hline 4 & Slab surface temperature & ${ }^{\circ} \mathrm{C}$ & \\
\hline 5 & ..... & & \\
\hline 1 & Plate number & - & \multirow{5}{*}{$\begin{array}{c}\text { Mill PCS } \\
->\text { Cooling } \\
\text { PCS }\end{array}$} \\
\hline 2 & Plate steel grade & - & \\
\hline 3 & Plate thickness & $\mathrm{mm}$ & \\
\hline 4 & Plate end rolling temperature & ${ }^{\circ} \mathrm{C}$ & \\
\hline 5 & $\ldots \ldots$ & & \\
\hline 1 & Plate target thickness & $\mathrm{mm}$ & \multirow{4}{*}{$\begin{array}{l}\text { Mill PCS -> } \\
\text { Gauge meter } \\
\quad \text { system }\end{array}$} \\
\hline 2 & Plate steel grade & - & \\
\hline 3 & Plate chemical composition & $\%$ & \\
\hline 4 & f.... & & \\
\hline
\end{tabular}

Socket method is used for mill PCS communication with other systems. According to the standard communication protocol, the address and port are used to distinguish the different parts of communication, and byte stream is used for transferring data information. According to mutually agreed way, both parts format and interpret data, and programming respectively. Socket method is difficult to implement, but it has higher real-time performance and flexibility[5]. Communication between mill PCS and other systems just has high demanding on speed, but less demanding on quantity.

Communication Application. The communication of plate mill PCS has been successfully applied in many plate plants such as TISCO and WISCO. The mill PCS is connected with other systems stably and all PCS functions are running well.

\section{Conclusions}

Data communication of PCS for the plate mill is realized. PCS and BAS is connected based on the industry Ethernet. BAS sends rolling actual data information to PCS periodically, and PCS sends setup data to BAS according to the trigger events. The communication between PCS and HMI is based on OPC protocol, and data tags are used. HMI sends operator intervention data to PCS, and PCS sends displaying information to HMI. Mediation tables are used for database communication between PCS and MES. With socket method, plate mill PCS is connected with furnace PCS, control cooling PCS and gauge meter system. With these methods, the plate mill PCS is communicated with related systems.

\section{Acknowledgements}

This work was financially supported by the Fundamental Research Funds for the Central Universities (N120407007)

\section{References}

[1] A. Nystrom, F. Agensjo, M. Smith, High performance information management in the rolling industry, Steel Times International, 19(1995) 17.

[2] Z. J. Jiao, Q. Zhang, C. Y. He, H. Zhang, Information Date Exchange for Plate Production Line Base on Database, Journal of Iron and Steel Research, 25(2013) 12-15. 
[3] C. Y. He, D. Wu, J. Wang, Study of Communication between S7-400 PLC and process control computer, Industry Control Computer 18(2005), 69-70.

[4] Z.P. Zhang, Oracle database application in remote data communication at Baosteel, Baosteel Technology, 2002, 2, 39-43.

[5] Y. Li, C. Y. Geng, Data synchronization over different platforms under module of socket communication, Journal of Beijing Institute of Technology, 22(2002) 81-84. 